\section{VISADOS DORADOS PARA INVERSORES EN ESPAÑA Y PORTUGAL: RESIDENCIA A CAMBIO DE DINERO}

\author{
Fernando Ampudia de Haro \\ Universidade Europeia \\ Centro de Investigação e Estudios de Sociologia-Instituto \\ Universitário de Lisboa (CIES-IUL) \\ ORCID iD: https://orcid.org/0000-0001-6470-1912 \\ fernando.ampudia@universidadeeuropeia.pt \\ Sofia Gaspar \\ ISCTE-Instituto Universitário de Lisboa (IUL) / Centro de \\ Investigação e Estudios de Sociologia-Instituto Universitário de \\ Lisboa (CIES-IUL) \\ ORCID iD: https://orcid.org/0000-0003-0002-6246 \\ sofia.gaspar@iscte-iul.pt
}

Cómo citar este artículo/Citation: Ampudia de Haro, F. y Gaspar, S. (2019). Visados dorados para inversores en España y Portugal: residencia a cambio de dinero. Arbor, 195 (791): a495. https://doi.org/10.3989/arbor.2019.791n1008

Recibido: 26 julio 2017. Aceptado: 8 noviembre 2018.

RESUMEN: La Autorización de Residencia para Inversores (ARI) o visado dorado en su denominación coloquial es una iniciativa que posibilita la adquisición del estatuto de residente a cambio de una inversión en el país de acogida. Portugal, desde 2012, y España, desde 2013, ofrecen este programa a quien lo desee y reúna las condiciones exigidas. El objetivo de este artículo es desarrollar un análisis comparado de los visados dorados en los dos países concretado en cuatro momentos diferentes. Inicialmente se ofrece una caracterización de la medida y sus requisitos y se describe su evolución. A continuación, se aborda su conceptualización sociológica a través de la noción de migraciones de capital y más concretamente como un fenómeno a medio camino entre la migración de negocios y la inversión externa extranjera. Después analizamos la racionalidad política que estructura este tipo de inversiones a partir del enfoque neofoucaltiano de la gubernamentalidad. Por último, abordamos las implicaciones cívicas y científicas de los visados dorados.

PALABRAS CLAVE: Autorización de Residencia para Inversores (ARI) o visados dorados; crisis económico-financiera; gubernamentalidad; España; Portugal.

\section{GOLDEN VISA FOR INVESTORS IN SPAIN AND PORTUGAL: RESIDENCE IN EXCHANGE OF MONEY}

Copyright: (C) 2019 CSIC. Este es un artículo de acceso abierto distribuido bajo los términos de la licencia de uso y distribución Creative Commons Reconocimiento 4.0 Internacional (CC BY 4.0).

ABSTRACT: Residence Permit for Investment Activities (ARI), colloquially known as golden visa, is an initiative that enables the acquisition of residency in exchange for a financial investment in the host country. Portugal, since 2012, and Spain, since 2013 , have offered this programme. The aim of this paper is to develop a comparative analysis of the golden visa at four different times. Firstly, we will characterize the measures, requirements and evolution of the programme. We will then conceptualize it sociologically using the notion of capital-linked migration and more specifically addressing it as a phenomenon half way between business migration programmes and foreign investment. Afterwards, we will analyze the political rationality that structures this initiative by using the neofoucaltian perspective of governmentality. Finally, we will address the civic and scientific implications of the golden visa.

KEYWORDS: Residence Permit for Investment Activities (ARI) or golden visa; financial economic crisis; governmentality; Spain; Portugal. 


\section{INTRODUCCIÓN}

El 11 de junio de 2013 el presidente del gobierno español, Mariano Rajoy, presentaba el anteproyecto de la ley de emprendedores y su internacionalización. Lo hacía en un acto organizado en el palacio de la Moncloa, junto a varios ministros y medio centenar de empresarios. Una de las medidas incluía la autorización de residencia a extranjeros que realizasen inversiones en el país comprando deuda, creando empleo o adquiriendo inmuebles. Por primera vez aparecían ante la opinión pública los popularmente conocidos como visados dorados, esto es, la posibilidad de residir en España a cambio de al menos una de aquellas inversiones. Unos meses antes, el 29 de enero, el ministro de Asuntos Exteriores portugués, Paulo Portas, entregaba la primera autorización de residencia del país vecino. Portugal había aprobado la medida en octubre de 2012 y el visto gold se concedía durante una ceremonia privada en el Palácio das Necessidades. Allí, el empresario indio Nesamanimaran Muthu recibía su autorización para residir en Portugal, tras haber formalizado la compra de tres hoteles en la región del Algarve.

Con poco tiempo de diferencia los gobiernos de ambos países ponían en marcha la Autorización de Residencia para Inversores (ARI) o visado dorado en su denominación coloquial, una iniciativa novedosa en el espacio ibérico ${ }^{1}$. Los visados dorados aúnan diversos componentes que los convierten en un objeto de interés para la sociología. Por un lado, el factor de la inversión externa y su relevancia para España y Portugal como vía de recuperación y salida de la crisis económico-financiera. Por otro, la posibilidad de desencadenar movimientos migratorios relacionados con el incentivo de la residencia. Un objeto, pues, del que poco se sabe, relativamente esquivo y con un período de vida aun hoy bastante limitado.

El objetivo de este artículo es realizar una aproximación general al fenómeno de los visados dorados en España y Portugal, considerando tanto sus implicaciones científicas como sociopolíticas. Esta aproximación no constituye una evaluación a partir de la cual justificar unívocamente la pertinencia o la eficacia de la medida. Antes bien, nuestra propuesta pasa por un análisis en profundidad de la naturaleza conceptual del fenómeno y de su legitimación económico-política, además de apuntar sus posibles repercusiones no solo en clave de conocimiento sociológico sino también en un sentido cívico. De acuerdo con tal objetivo nuestro trabajo se abre (sección 2) con una caracterización de la Autorización de Residencia para Inversores, identifi- cando sus requisitos de forma comparada en el ámbito internacional para, desde ahí, ofrecer una descripción de su evolución en España y Portugal. A continuación (sección 3), consideramos la dimensión conceptual de la ARI a través de la noción de migraciones de capital (capital linked migration) (Tseng, 2000), lo que nos permite pensarla en una suerte de interregno entre los programas de migración de negocios (business migration programmes) y la inversión extranjera directa (foreign direct investment). Después (sección 4) analizamos la racionalidad política que la estructura a partir del enfoque neofoucaltiano de la gubernamentalidad. De este modo, accedemos a los principios que justifican la medida, su alineamiento con los objetivos generales de gobierno y la noción de sujeto que demanda y simultáneamente desea moldear. Por último (sección 5), abordamos las implicaciones cívico-políticas y sociológicas de los visados dorados. Las primeras derivan bien de la mayor o menor disociación entre la residencia y la noción de ciudadanía, bien de la posibilidad de entender esta última desde una perspectiva exclusivamente económica. Las segundas se centran en las opciones que pueden o no abrirse, a partir de este tipo de permiso de residencia, para el estudio sociológico de las migraciones. Finalizamos con un apartado dedicado a las conclusiones, necesariamente abierto a futuras investigaciones.

\section{CARACTERIZACIÓN Y EVOLUCIÓN DE LOS VISADOS DORADOS}

Los visados dorados en la ley portuguesa y española no presentan diferencias substanciales. Existen algunas especificidades que los distinguen, aunque lo más reseñable sea constatar que Portugal avanzó un año antes con la medida. La Autorização de Residência para Atividade de Investimento entró en vigor el 8 de octubre de 2012 (Portaria no 305-A/2012, de 4 de Outubro) ${ }^{2}$.

En Portugal la ARI puede ser obtenida de acuerdo con los siguientes tipos de inversión:

a) Transferencia de capitales por un montante igual o superior a un millón de euros.

b) Creación de al menos diez puestos de trabajo.

c) Adquisición de bienes inmuebles por un montante igual o superior a 500.000 euros.

d) Adquisición de bienes inmuebles destinados a rehabilitación urbana por un montante igual o superior a 350.000 euros.

e) Transferencia de capitales por un montante superior a 350.000 euros cuya aplicación se destine a 
actividades de investigación en instituciones del sistema científico nacional.

f) Transferencia de capitales por un montante igual o superior a 250.000 euros dedicados al apoyo de actividades artísticas o a la recuperación y mantenimiento del patrimonio cultural.

g) Transferencia de capitales por un montante igual o superior a 500.000 euros en fondos de inversión o capital de riesgo con el objetivo de recapitalizar pequeñas y medianas empresas.

Una vez cumplidos los requisitos exigidos, la ARI en Portugal ofrece los siguientes derechos:

a) Entrada en el país sin necesidad de visado de estancia.

b) Residir y trabajar cumpliendo el requisito mínimo de permanencia de siete días durante el primer año y no menos de catorce en los años siguientes. Concretamente, la autorización se concede inicialmente por un año, pero puede renovarse por dos periodos consecutivos de dos años. Es precisamente en estos períodos adicionales cuando se exige un total mínimo de catorce días de permanencia. Por lo tanto, el solicitante que desee agotar su ARI deberá garantizar al menos 35 días de permanencia en el país durante cinco años.

c) Circular por el espacio Schengen sin necesidad de visado.

d) Reagrupamiento familiar (cónyuges e hijos).

e) Solicitar la Autorización de Residencia Permanente. Se puede acceder a ella después de cinco años de residencia temporal o tras las dos renovaciones que permite la ARI.

f) Adquirir la nacionalidad portuguesa tras cinco años de residencia.

Por su parte, en España habría que esperar hasta la ley 14/2013, de 27 de septiembre, la denominada ley de apoyo a los emprendedores y su internacionalización, para recoger la normativa relacionada con el visado de residencia para inversores, emprendedores, profesionales altamente cualificados, traslado intraempresarial e investigadores. Entre su promulgación y el 30 de junio de 2018, dicha ley ha captado un total de 13.522 autorizaciones por motivos económicos, de las cuales $6.446(47,7 \%)$ corresponden a profesionales altamente cualificados, $3.621(26,8 \%)$ a inversores, $1.727(12,8 \%)$ a trasladados intraempresariales, 1.437 $(10,6 \%)$ a investigadores y 291 (2,2\%) a emprendedores (véase el informe Extranjeros residentes en Espa- ña. A 30 de junio de 2018. Principales Resultados). Entre estas categorías solo la de inversor es equiparable a los supuestos que permiten acceder a la ARI en Portugal. Por ese motivo, será la única que consideremos de ahora en adelante.

En el caso de los inversores las vías de acceso a la ARI española son las siguientes:

a) Inversión por un montante igual o superior a dos millones de euros en títulos de deuda pública, o de un millón de euros o más en acciones y participaciones de empresas nacionales, o de un millón de euros en depósitos bancarios.

b) Adquisición de bienes inmuebles por un montante igual o superior a 500.000 euros.

c) Presentación de proyectos empresariales que puedan ser considerados de interés general.

d) Declaración de intención de desarrollo de una actividad emprendedora de carácter innovador, cuya tramitación y planificación requieran permanecer en el país durante un año antes de su puesta en marcha y que, a su vez, cuente con un informe favorable de la oficina comercial donde se presenta la solicitud.

En España los derechos que confiere la ARI son prácticamente similares a los de Portugal, si bien en este punto pueden apreciarse algunas diferencias:

a) Viajar o circular sin visado por el espacio Schengen.

b) Reagrupamiento familiar (cónyuges e hijos).

c) La autorización de residencia inicial es por un período de dos años, permitiéndose la renovación por periodos de dos años. La renovación no exige ningún tipo de visita o permanencia en el país. Antes de la renovación es necesario visitar España al menos una vez al año.

d) Entrada en el país sin necesidad de visado de estancia.

e) Solicitar la Autorización de Residencia Permanente de acuerdo con la preceptiva legal.

f) Adquirir la nacionalidad española tras diez años de residencia.

Conviene señalar que en los dos casos la ARI ha sufrido alteraciones con relación a la versión inicial. Esas alteraciones tienen como objetivo limar aspectos que puedan desincentivar a los potenciales solicitantes. Pese a que los requisitos principales se mantienen, es cierto que se han ampliado los supuestos de inversión 
(es el caso de las actividades artísticas o la rehabilitación urbana en Portugal), se han rebajado los plazos de permanencia, se han ampliado los beneficiarios (parejas de hecho en el caso español) o se han simplificado los trámites administrativos. En este sentido la ARI está sujeta a un cierto dinamismo dependiente a su vez de aquellas mejoras que puedan suscitar el interés del solicitante ${ }^{3}$.

A continuación analizaremos con mayor detalle la evolución de ambos programas en lo que se refiere a solicitudes y volumen de inversión captado. En este sentido, es importante destacar que los datos existentes no permiten todavía un nivel de desagregación profundo. Es posible ofrecer una panorámica general, en el caso portugués, a partir de los datos del Serviço de Estrangeiros e Fronteiras y en el caso de España, empleando los datos procedentes del Ministerio de Trabajo, Migraciones y Seguridad Social.

Tabla 1. Evolución de las autorizaciones de residencia para inversores en Portugal

\begin{tabular}{|c|c|c|}
\hline & TOTAL & 6813 \\
\cline { 2 - 3 } Número de & 2012 & 2 \\
autorizaciones & 2013 & 494 \\
de residencia & 2014 & 1526 \\
& 2015 & 766 \\
& 2016 & 1414 \\
& 2017 & 1351 \\
Principales & 2018 & 1260 \\
\hline nacionalidades & China & 4031 \\
& Burquía & 625 \\
& Sudáfrica & 279 \\
\hline Total inversión & Rusia & 268 \\
captada & 4.155.454.320,27 & ----- \\
\hline
\end{tabular}

Fuente: Autorização de residencia para atividade de investimento (ARI). Dados de 08 de outubro de 2012 a 30 de novembro de 2018. Serviço de Estrangeiros e Fronteiras.

La tabla 1 nos muestra que, en total, fueron emitidos, hasta noviembre de 2018, 6.813 golden visa en Portugal. La expedición de visados crece hasta 2014, disminuyendo en 2015 con la interrupción del programa, debido a un caso de corrupción. Tras reanudarse, se produce un aumento en 2016 y una ligera bajada en 2017 (las cifras de 2018 todavía son provisionales). En lo que toca al total de inversión captada, los datos oficiales se sitúan en torno a los 4.155.454.320,27 millones de euros, la mayor parte procedentes de la opción inmobiliaria. Si nos detenemos en las cinco nacionalidades que copan el mayor número de solicitudes, constatamos que el programa está dominado principalmente por ciudadanos chinos (4.031) y brasileños (625). En la tabla 2 observamos que el $94,5 \%$ de los ingresos deriva de la compra de casas y tan solo un $5,2 \%$ de transferencias directas de capital. Por su parte, la creación de puestos de trabajo registra un valor residual, suponiendo el $0,17 \%$ de la inversión.

En el caso de España las series estadísticas sobre "extranjeros con certificado de residencia o tarjeta de residencia en vigor" procedentes del Ministerio de Trabajo, Migraciones y Seguridad Social, arrojan semestralmente registros sobre inversores al abrigo de la ley 14/2013. La tabla 3 muestra que entre 2014 y el 30 de junio de 2018 el número se sitúa en 9.674 personas. Se constata que desde su comienzo el número de inversores ha aumentado de 531 personas en 2014 a 3.621 en 2018 . Asimismo, verificamos que el número de autorizaciones en España (9.674) es superior al de Portugal (6.813)

Más allá de esta panorámica general, el único informe que recoge información desagregada sobre los visados dorados en España es de abril de 2015. En este documento (Informe sobre la aplicación de la Sección de Movilidad Internacional de la Ley 14/2013, de 27 de septiembre, de apoyo a los emprendedores y su internacionalización), los datos se refieren exclusivamente al periodo comprendido entre el inicio del programa (30 de octubre de 2013) y el 31 de diciembre de 2014 (tabla 4). En consecuencia, solo es posible comparar parcialmente algunas categorías entre ambos países.

Se observa que entre 2013 y 2014 los visados dorados generaron una inversión cercana a los 447 millones de euros. Para este montante la tendencia que se detecta es similar a la portuguesa: la opción inmobiliaria logra captar aproximadamente el 93\% del total, siendo residuales la transferencia de capital $(5,46 \%)$ y la creación de empleo (2,26\%).

En la tabla 3, comprobamos que las nacionalidades que predominan son ligeramente diferentes a las existentes en Portugal, si bien los ciudadanos chinos son igualmente quienes más invierten, con un total de 3.089 visados, seguidos de los ciudadanos de origen 
Tabla 2. Criterios de concesión de autorizaciones de residencia para inversores en Portugal

\begin{tabular}{|c|c|c|c|c|}
\hline \multirow{4}{*}{ Por criterio } & & $\mathbf{N}$ & $\%$ & $€$ \\
\hline & Inversión inmobiliaria & 6441 & 94,5 & $3.769 .059 .383,67$ \\
\hline & Transferencia capital & 360 & 5,2 & $386.394 .936,60$ \\
\hline & Creación empleo & 12 & 0,17 & ----------- \\
\hline
\end{tabular}

Fuente: Autorização de residencia para atividade de investimento (ARI). Dados de 08 de outubro de 2012 a 30 de novembro de 2018. Serviço de Estrangeiros e Fronteiras.

Tabla 3. Evolución de las autorizaciones de residencia para inversores en España

\begin{tabular}{|c|c|c|}
\hline & TOTAL & 9674 \\
\cline { 2 - 3 } & 2014 & 531 \\
Número de autorizaciones de & 2015 & 803 \\
residencia para inversores & 2016 & 1814 \\
& 2017 & 2905 \\
\hline Principales nacionalidades & 2018 & 3621 \\
\hline (2015-2017) & China & 3089 \\
\hline
\end{tabular}

Fuente: Extranjeros residentes en España. A 30 de junio de 2018. Principales Resultados. Ministerio de Trabajo Migraciones y Seguridad Social.

Tabla 4. Criterios de concesión de autorizaciones de residencia para inversores en España

\begin{tabular}{|c|c|c|c|c|}
\hline \multirow{4}{*}{ Por criterio } & & $\mathbf{N}$ & \% & $\boldsymbol{\epsilon}$ \\
\cline { 2 - 5 } & Inversión inmobiliaria & 490 & 92,28 & $369.766 .625,00$ \\
\cline { 2 - 5 } & Transferencia capital & 29 & 5,46 & $37.543 .696,00$ \\
\cline { 2 - 5 } & Creación empleo & 12 & 2,26 & $395.336,00$ \\
\hline
\end{tabular}

Fuente: Informe sobre la aplicación de la Sección de Movilidad Internacional de la Ley 14/2013, de 27 de septiembre, de apoyo a los emprendedores y su internacionalización. Abril 2015. Ministerio de Empleo y Seguridad Social, Ministerio de Asuntos Exteriores y Cooperación, Ministerio de Economía y Competitividad, Ministerio del Interior.

ruso con 2.618 visados (véase Extranjeros residentes en España. A 30 de junio de 2018. Principales Resultados).

Merece la pena detenernos un momento en la presencia de ciudadanos chinos en España y Portugal. En sendos países esta comunidad es la quinta más numerosa entre el total de los residentes extranjeros: 211.298 individuos (3,9\%) en España y 23.197 en Portugal (5,5\%) (véanse Instituto Nacional de Estadística, 2017; Relatório de Imigração, Fronteiras e Asilo, 2017). En ambos países, los inmigrantes chinos son fundamentalmente inmigrantes laborales, aunque su procedencia social se haya ido diversificando en los últimos años con la llegada de profesionales cualificados y de estudiantes universitarios (Gaspar, 2017). Es necesario tener en cuenta que el paulatino aumento de ciudadanos chinos al abrigo de los visados dorados corre en paralelo al incremento de la inversión externa china en las economías ibéricas, especialmente, en Portugal.

\section{LOS VISADOS DORADOS ENTRE LA MIGRACIÓN DE CAPITAL Y LA INVERSIÓN EXTRANJERA}

El fenómeno de los visados dorados puede ser entendido conceptualmente a partir de una doble lógica:

a) La lógica de los patrones migratorios no convencionales. Identificamos como convencionales 
aquellos tipos de movilidad asociados a mano de obra que se desplaza hacia zonas excedentarias en capital, pero deficitarias en fuerza de trabajo (inmigración laboral). La movilidad vinculada a la ARI no responde a este modelo. Antes bien, se trata de desplazamientos de individuos provistos de capital hacia zonas deficitarias precisamente en capital.

b) La lógica de la internacionalización de los flujos de capital. Estos circulan en el marco de una economía globalizada y sometida a condiciones de competencia donde los estados pugnan por captar inversión.

Esta doble lógica aparece incorporada en la idea de migración de capital (capital linked migration) que, de acuerdo con Tseng (2000), puede desdoblarse en dos componentes principales:

a) Programas migratorios de negocios (business migration programes), con el propósito de atraer inmigrantes que posean un valor económico diferencial por sus cualificaciones, por su potencial de inversión o por su capacidad para generar nuevos empleos. Canadá, Estados Unidos, Taiwán, Corea del Sur o Australia son países que típicamente aplican tales programas facilitando o simplificando la condición de residente.

b) Inversión directa extranjera (foreign direct investment). Supone la colocación de un determinado volumen de capital en una economía receptora creando nuevas estructuras empresariales o participando en las ya existentes. La llegada de este capital puede venir o no acompañada de inversores que decidan fijar la residencia en ese nuevo espacio. Esto es, se trata de un tipo de inversión que hipotéticamente podría desencadenar procesos migratorios, si bien no los determina obligatoriamente.

Entendemos que la ARI española y la portuguesa se identifican con ambos componentes: en los dos casos comporta una inversión que podría venir acompañada de movilidad y sedentarización. Aquí el factor residencia es a priori un factor no garantizado; es decir, no tiene por qué producirse. Más adelante veremos que esa falta de garantías es contrarrestada por indicios que permiten pensar en la inversión y en la migración como factores estrechamente relacionados. Sin embargo, antes de llegar a ese punto, resulta lógico pensar que dicha falta de garantías deriva en gran medida de uno de los rasgos más paradójicos de los golden visa: se autoriza la residencia al inversor sin que se le exija residir o este tenga intención real de hacerlo. A lo sumo, en Portugal, pasar 35 días en cinco años, bastando en España una simple visita al país.

Los visados dorados operan bajo las dinámicas que se establecen para atraer propietarios de capital. Si España y Portugal compiten contra otros muchos países por hacerse con volúmenes significativos de inversión, no es menos cierto que esa competencia también se instituye internamente en el marco peninsular. En términos genéricos, la atracción de inversores-migrantes se articula en torno a una serie de valores y promesas fundamentales:

a) Seguridad legal y administrativa para las inversiones extranjeras. Estabilidad política y previsibilidad como vías de reducción de la incertidumbre y del riesgo.

b) Entorno favorable a la recepción de inversiones: las condiciones que ofrece el país permiten la reproducción de la inversión y aseguran rentabilidad.

c) Fiscalidad ventajosa y simplificación de trámites administrativos.

d) Regulación clara que elimina la arbitrariedad legal, así como los controles y las inspecciones abusivas.

e) Posibilidad de acceso al espacio Schengen y a otros mercados europeos.

f) Condiciones de vida favorables relacionadas con el clima, la educación, la seguridad física o la cultura.

Junto a esta dinámica competitiva de carácter global existen factores coyunturales relacionados con situaciones históricas concretas. España y Portugal, a raíz de la crisis financiera iniciada en los Estados Unidos en 2008 y transmutada en Europa como crisis de las deudas soberanas a partir de 2010-2011, han vivido y continúan viviendo graves problemas económicos y financieros. Tales problemas han merecido como respuesta las denominadas políticas de austeridad (Blyth, 2013) que, en lo que toca al asunto que abordamos, colocan el acento en la reducción del gasto público y en la liberalización y privatización de bienes y servicios como medios de recuperación y aumento de la competitividad. Así pues, en una coyuntura de recortes presupuestarios la atracción de inversión extranjera ha adquirido una notable transcendencia como medio de obtención de recursos. Esto es, la emergencia de la ARI española y de la portuguesa difícilmente podrá comprenderse si no se inscribe en este contexto de crisis. 
En este mismo contexto, es necesario reflexionar brevemente sobre el papel de la Unión Europea en relación con las políticas de los golden visa. En este dominio no existe una política concertada por parte de la Unión Europea, una vez que en general se entiende que estos programas forman parte de las iniciativas particulares de los estados miembros para captar inversión extranjera. En ese sentido los países se desenvuelven en un mercado competitivo intraeuropeo, en el que ofrecen condiciones más o menos ventajosas en relación con sus competidores. Hasta el momento la Unión Europea solo se pronuncia o ha pronunciado en dos situaciones específicas: a) cuando los países ofrecen directamente la nacionalidad a los inversores extranjeros a cambio de montantes considerados improcedentes ${ }^{4}, \mathrm{o}$ b) cuando reclama precaución y fiscalización de los flujos de dinero entrantes a través de los programas ARI, para evitar que se convierta en un sistema de blanqueo de capitales (Carrera, 2014).

\section{LOS VISADOS DORADOS COMO MATERIALIZACIÓN DE UNA RACIONALIDAD POLÍTICA NEOLIBERAL}

Una vez caracterizados legal, conceptual y evolutivamente los visados dorados, es el momento de analizar el tipo de racionalidad política presente en esta medida. Para ello, adoptamos una perspectiva propia de los estudios sobre gubernamentalidad, de inspiración neofoucaltiana (Ampudia de Haro, 2006; Ampudia de Haro, 2013; Ampudia de Haro, 2014; Burchell, Gordon y Miller, 1991; Marinis, 1999). El concepto de "racionalidad política" alude al conjunto de objetivos que se pretende alcanzar en el ejercicio del poder, así como a los principios que se esgrimen para legitimar dichos objetivos. Estos pueden ser de naturaleza diversa (política, económica, social, cultural, educativa, militar), sucediendo exactamente lo mismo con dichos principios (libertad, equidad, justicia, prosperidad). Cada variante de racionalidad política maneja una noción concreta de los individuos que son objeto de gobierno y expresa sus objetivos en articulación con los medios para alcanzarlos de acuerdo con un discurso particular. Por lo tanto, esta conjunción entre objetivos, principios, visión de los individuos y discurso, en lo que toca a los visados dorados, constituye el eje de nuestro análisis.

De hecho, los golden visa son la expresión manifiesta de un tipo de racionalidad política neoliberal. Cuando se usa el término neoliberal, lo más frecuente es referirse a un conjunto de políticas públicas expresamente orientadas al mercado (Larner, 2000). No es este el sentido que empleamos aquí. Desde la óptica de los estudios sobre gubernamentalidad, el neoliberalismo como racionalidad política es un modo de gobierno que apuesta por economizar el ejercicio del poder. Esto implica que sean los propios individuos los que inviertan en su autogobierno con la máxima competencia e intensidad posibles. El ejercicio de este autogobierno supone concebir a los sujetos gobernados como sujetos dotados de autonomía, que deberá ser alineada con los objetivos globales que se persiguen para el gobierno de la sociedad.

Como hemos dicho anteriormente, en España y Portugal el contexto inmediato en el que emergen los visados dorados es definido por las políticas de austeridad. De acuerdo con Blyth (2013), estas políticas a) promueven intencionalmente procesos de deflación a través de los cuales b) la economía sufre un ajuste por medio de la reducción de salarios, precios y gasto público c) con el propósito de restablecer la competitividad económica d) la cual, gracias a la disminución del déficit y del endeudamiento público e) provocará un aumento de la confianza de los inversores, pues la contención impide que el estado acapare todas las posibilidades de financiación, f) lo que producirá una expansión de la actividad económica.

Se busca provocar un efecto expansionista y reactivador mediante la contracción de elementos centrales de la actividad económica, como el gasto público. En este cuadro de reducción del gasto público asociado a las políticas de bienestar y protección social los visados dorados se relacionan con una concepción económica de la ciudadanía (Dzankic, 2012). De acuerdo con esta concepción se considera aceptable la inclusión de individuos en una determinada comunidad si el coste de tal inclusión no resulta superior a los hipotéticos beneficios que dichos individuos generarán. Visto así, quien desea integrarse en la comunidad pero aumenta los costes derivados de esa integración, supongamos, en concepto de apoyos, subsidios, atención técnica e infraestructuras, supone un lastre para la propia comunidad, que ve crecer el coste de mantener su nivel de bienestar. Lo contrario, desde esta óptica, sería lo deseable: que la llegada de nuevos individuos reduzca el coste de mantenimiento del bienestar, es decir, que su aportación a la sociedad de acogida supere los costes de su presencia. Se trataría de individuos que no producen gastos para la comunidad, porque vienen acompañados de una determinada inversión: portan los medios para hacerse cargo de sí mismos, y en consecuencia no sobrecargan el sistema de bienestar en tiempos de reducción del gasto público. Como señalá- 
bamos, se optimiza el ejercicio del poder a través del autogobierno de los ciudadanos.

Al mismo tiempo los visados dorados revelan cómo el estado articula su política económico-migratoria con arreglo a la circulación mundial de capitales. Al contrario de lo que se suele pensar, el neoliberalismo no equivale simplemente a la sustitución del estado por el mercado. Entendido como racionalidad política, no preconiza estrictamente esa sustitución y sí la utilización del aparato estatal para la creación de mecanismos mercantiles que regulen la inversión asociada a la potencial llegada y permanencia de inversores. En este sentido, los golden visa se integran en un mercado de captación de inversores que institucional y legalmente es moldeado por los estados en condiciones de competencia. El concurso y la intervención estatal es, pues, imprescindible en la configuración del mecanismo mercantil que preside la oferta de autorizaciones de residencia.

La lógica mercantil, por extensión, justifica el carácter de excepcionalidad de los visados dorados. No olvidemos que otorga de forma rápida y accesible la posibilidad de residencia, con reducciones significativas de trámites administrativos y plazos, amén de opciones suplementarias como el reagrupamiento familiar o una ulterior naturalización. Dicho de otro modo, quienes no poseen capital para invertir están privados de esta vía preferente. Existe una categorización de los sujetos como especiales, excepcionales o prioritarios que convive con el principio convencional de la igual aplicación de la ley para todos los ciudadanos (Ong, 2006). Tal excepcionalidad se justifica en virtud del interés que despiertan los inversores y de la oferta de incentivos para retener las inversiones.

Cuenta además con su correspondiente legitimación técnico-científica. Un ejemplo de esta es el trabajo de Xu, El-Ashram y Gold (2015), auspiciado por el Fondo Monetario Internacional. Encontramos en este documento todo un inventario de argumentos orientados a un adecuado diseño de la estructura de mercado de los visados dorados. Tras identificar aquellos aspectos calificados como positivos -es decir, como inversiones dinamizadoras de la economía nacional, generadoras de empleo y potenciadoras de la recaudación fiscal-, los autores se detienen en aquellas dimensiones potencialmente negativas: concentración excesiva de la actividad económica en áreas relacionadas con la inversión, y por extensión falta de diversificación, alteraciones legales casuísticas que no favorecen la estabilidad de las inversiones, y por último el lavado o el ocultamiento de dinero de procedencia irregular o ilegal.
La solución a estos problemas pasa, como recomiendan los autores, por la gestión prudente de este instrumento de inversión, pero fundamentalmente por un diseño institucional del mercado de los visados dorados que garantice la eficiencia mediante la eliminación de asimetrías informativas. Es decir, el objetivo es que dicho mercado funcione adecuadamente según a) la dinámica de la oferta y la demanda, b) el precio como síntesis total de toda la información disponible para los competidores y c) el juego de incentivos ofrecidos. En consecuencia, reglas claras y transparencia informativa; esto es, la eliminación de distorsiones que puedan afectar a la competencia.

La transparencia se convierte en una garantía de que el mercado funcionará eficientemente según su propia dinámica competitiva. Por ese motivo, se conceptualiza como una suerte de criterio técnico que permite el desarrollo de la lógica mercantil. No obstante, también admite una conceptualización ética. Desde esta perspectiva, una gestión transparente del programa favorecería su aceptación por parte de la ciudadanía. Que los golden visa sean vistos como iniciativas legítimas dependerá de que no dañen la reputación del país (Xu, El-Ashram y Gold, 2015). Esa reputación podría resultar afectada por sospechas de que los visados dorados son un mecanismo que permite blanquear u ocultar dinero o que vulnera el principio de igualdad entre los ciudadanos. Frente al primer recelo, se recomienda la verificación de la identidad y el pasado de cualquier inversor, así como el establecimiento de procedimientos administrativos rigurosos. Frente al segundo, se recomienda informar a la opinión pública sobre el número de visados dorados concedidos y sobre el volumen de inversión captada. Así pues, la idea de transparencia funciona simultáneamente como criterio de eficiencia del mercado y como criterio de legitimación política.

El análisis teórico que hasta aquí se ha desarrollado encuentra su materialización empírica en el discurso de los responsables políticos de esta medida. Este discurso no difiere substancialmente entre los dos países, y globalmente reproduce la lógica anteriormente descrita. Comenzando con Portugal, es imprescindible recuperar la figura de Paulo Portas, Ministro de Estado y de Asuntos Exteriores (Negócios Estrangeiros) del XIX Gobierno Constitucional (2011-2015), en calidad de defensor y promotor público del programa. Sus intervenciones permiten ilustrar el tipo de justificación empleada mediante argumentos alusivos al sistema de competición global para atraer inversores. Tras un año de funcionamiento, Portas anunciaba que el pro- 
grama había logrado captar 306,7 millones de euros gracias a 471 visados, lo que excedía en 100 millones de euros las previsiones iniciales. Esto demostraba que el país, en su opinión, había ganado relevancia en el panorama internacional: “[...] es una señal muy práctica de que Portugal está de vuelta al GPS de los países en los que es interesante invertir" (véase "Paulo Portas diz que o programa que concede vistos 'gold' excedeu largamente o objetivo").

Sin embargo, este posicionamiento prometedor se resiente cuando en noviembre de 2014 se desencadena la operación laberinto y se detiene a once personas, incluyendo a altos funcionarios del estado, por sospechas de corrupción, tráfico de influencias y blanqueamiento de capitales en el proceso de adjudicación de los visados dorados. Esto motivó la dimisión del ministro de Administración Interna, Miguel Macedo, y la suspensión temporal del programa. El debate público y los medios de comunicación abordaron la cuestión de la reputación del país temiendo que el caso minase el valor de Portugal como espacio de inversión. El programa fue reactivado poco tiempo después y, gracias a la presencia de Paulo Portas en la comisión de investigación parlamentaria al hilo de aquella operación, en marzo de 2015 , contamos con algunas declaraciones justificativas de la medida:

a) La competencia global por la atracción de inversores "[...] va a permanecer; es una realidad indiscutible" (véase en Público: «Paulo Portas: "Estamos disponíveis para melhorar sistema" dos vistos gold»).

b) Renunciar a esta posibilidad de inversión es un gesto "insano" toda vez contribuye a la generación de riqueza. En el caso del sector inmobiliario, "las casas no caen del cielo, alguien tiene que construirlas [...] esto deja impuestos en los ayuntamientos y en el estado portugués" (véase "Vistos Gold. "Não é por haver uma fraude numa avaliação que se acaba com os exames", diz Portas»).

c) Cualquier fragilidad o titubeo redunda en un avance de los competidores: "cuando el programa portugués tuvo un periodo de alguna incertidumbre, el mercado que creció fue el español" (véase «Paulo Portas: "Estamos disponíveis para melhorar sistema" dos vistos gold»).

El cambio de gobierno no supuso ninguna alteración en el programa. EI XXI Gobierno Constitucional, encabezado por el Partido Socialista y con apoyo parlamentario del Bloco de Esquerda y del Partido Comunista, ha dado continuidad a la iniciativa haciendo un balance positivo de su ejecución (véase "António Cos- ta destaca política de alargamento dos "vistos gold" em visita à China"; "Vistos gold: Quatro anos são positivos mas programa está longe de ser principal arma para captar investimento").

Como señalamos anteriormente, en España el tipo de discurso de los responsables políticos es similar. Soraya Sáenz de Santamaria, vicepresidenta del gobierno, desgranaba algunos argumentos en abril de 2013 tras la aprobación del anteproyecto de la ley de emprendedores, donde se enmarcan los visados dorados:

- Necesidad de posicionar el país en el mercado global de captación de inversión: "Lo que hacemos es, ni más ni menos, lo que están haciendo otros estados de nuestro entorno para atraer inversores" (véase "Los extranjeros obtendrán la residencia al comprar un inmueble de más de $500.000 €^{\prime \prime}$ ).

- Los inversores tienen un efecto positivo en la economía nacional: "están contribuyendo a la riqueza del país y se ha considerado oportuno que obtengan la residencia de manera ordenada y disciplinada" (véase "Los extranjeros obtendrán la residencia al comprar un inmueble de más de $\left.500.000 €^{\prime \prime}\right)$.

Sin embargo, antes de la aprobación definitiva del programa fue posible asistir al debate sobre el montante mínimo de la inversión requerida para obtener la ARI. Si bien se trató de una controversia con escasa repercusión en los medios de comunicación, permite contemplar una parte del proceso que instituye el mercado de los visados dorados gracias al concurso fundamental del estado. Un año antes el Secretario de Estado de Comercio, Jaime García-Legaz, situaba en 160.000 euros ese montante mínimo; un montante de equilibrio entre el incentivo a una llegada excesiva de solicitantes y las necesidades de financiación del país: "No se puede poner un límite de precio más bajo porque generaría una demanda masiva de permisos de residencia. Un precio inferior podría servir de excusa para obtener la residencia" (véase "El Gobierno concederá la residencia a los extranjeros que compren una casa por más de 160.000 euros").

Lo que está en juego es el coste reputacional: si el requisito de inversión es excesivamente bajo, la información que se transmite al mercado es la de un país que vende abiertamente y sin tapujos la residencia. Así lo interpretaba una consultora especializada en el mercado chino tiempo después de haber sido establecido el montante mínimo en 500.000 euros: "El precio anterior [160.000 euros] no daba una buena imagen 
y creaba un clima de desconfianza y confusión" (Cabrerizo, 2013, 29 abril)5. La razón de ser de aquella cantidad, según la prensa económica especializada, guardaría relación con el "[...] prototipo de inmueble predominante, en torno a los 160.000 euros de valor" (Soler-Matutes y Lijing, 2012, 23 noviembre). Es decir, la variable inmobiliaria adquiere una trascendencia definitiva, como apunta el propio presidente del Gobierno, Mariano Rajoy, al hablar de la necesidad de vender las casas derivadas de la burbuja vivida por el sector (véase "Rajoy sobre la residencia para compradores: 'Tenemos que vender stock'”).

Ni Portugal ni España divergen, por lo tanto, en el modo de pensar, justificar y legitimar los visados dorados: la racionalidad política neoliberal define en los dos casos la substancia de la medida.

\section{LAS IMPLICACIONES CÍVICO-POLÍTICAS Y SOCIO- LÓGICAS DE LOS VISADOS DORADOS}

Los programas ARI originan diversas reflexiones relacionadas con el concepto de 'ciudadanía' (Dzankic, 2012; Peláez-Paz y Sanz Abad, 2014). No pretendemos ser exhaustivos en este punto, por lo que nos limitamos a apuntar las líneas maestras de los problemas que los golden visa plantean en el ámbito cívicopolítico y sociológico.

El concepto de ciudadanía alude al tipo de relación que se establece entre el individuo y el estado, mediada por una serie de derechos y deberes recíprocos. De modo sintético, las principales dimensiones de la ciudadanía son las siguientes:

a) Voluntad y posibilidad de participación en las decisiones relativas a los asuntos de la comunidad.

b) Protección del ciudadano a través de la ley con obligación de su cumplimiento.

c) Igualdad de todos los ciudadanos en el ejercicio de sus derechos y deberes legales.

d) Legitimidad de las instituciones que garantizan los derechos y deberes de los ciudadanos.

e) Sentido de pertenencia a una comunidad asociado al vínculo de la residencia.

f) Lealtad a las instituciones estatales-nacionales por parte de los ciudadanos.

Convencionalmente el concepto de ciudadanía y el hecho de ser considerado un ciudadano son dos elementos relativamente independientes de las condiciones materiales poseídas por cada individuo, una vez que la ciudadanía se construye al margen del estatuto económico de la persona. Sin embargo, los visados dorados revelan cómo algunas de las dimensiones de la ciudadanía -en este caso la residencia- se articulan alrededor del mercado, disociándose del territorio y de la permanencia física y estructurándose en función de la circulación global de flujos de capital (Ong, 2005; Ong, 2006). Como apuntan Walsh (2011) y Peláez-Paz y Sanz Abad (2014), con los golden visa asistimos a modulaciones de la ciudadanía en función de las dinámicas de dichos flujos.

Paralelamente la cuestión que emerge es la posibilidad de instrumentalizar dimensiones de la ciudadanía ofrecidas ahora como ventaja competitiva en línea con una concepción económica del ciudadano (Shachar y Bauböck, 2014). El acceso diferencial y preferente a la residencia, que a su vez podría abrir el camino a la naturalización y por ende a la ciudadanía plena, contradice una noción de 'ciudadano no sujeto a evaluaciones económicas como fundamento de su condición'.

Esa instrumentalización obviamente coloca bajo sospecha al beneficiario de los golden visa. Su vinculación a la comunidad puede leerse en clave exclusivamente utilitaria con el consiguiente riesgo para la cohesión de la sociedad: la falta de compromiso o identificación atribuida a sus beneficiarios resulta especialmente relevante en momentos de crisis colectiva, cuando difícilmente podrá apelarse a ellos para que, junto al resto de la ciudadanía, realicen los esfuerzos o incluso los sacrificios necesarios para superar esas dificultades (Shachar, 2014). Este punto resulta esencial en España y Portugal, donde la virulencia de la crisis económico-financiera iniciada en 2008 se ha traducido en pérdidas significativas de poder adquisitivo, pauperización, desempleo y desprotección social entre amplias capas de la población. Al tiempo, las políticas de austeridad han venido acompañadas de un discurso legitimador que enfatiza, precisamente, la idea de sacrificio, de frugalidad y de esfuerzo colectivo para salir de una coyuntura social exigente y dura (Alonso, Fernández Rodríguez e Ibáñez Rojo, 2016). Existe, por lo tanto, ese riesgo de desmoralización de la ciudadanía ante medidas percibidas como no igualitarias, no equitativas y cívicamente poco ejemplares.

Por otra parte, que estos programas puedan ser objeto legítimo de estudio dentro del campo de la sociología de las migraciones es una cuestión discutible. En última instancia, los visados dorados pueden concederse a quien ni siquiera reside, vaya a residir, o tenga intención de residir en el país en el que realiza la inversión. Esto es, puede no estar aso- 
ciada a ningún tipo de movilidad o permanencia. En este sentido no dejaría de ser una variante más de inversión extranjera directa que intenta ser atraída con ayuda de incentivos y ventajas para el inversor. Paradójicamente, como ya apuntamos anteriormente, se otorga la posibilidad de residencia sin que residir o permanecer sean requisitos determinantes. Sin movilidad ni permanencia, restarían apenas desplazamientos puntuales del titular del golden visa para observar y controlar el estado de sus inversiones. Desde esta óptica, estos programas difícilmente podrían relacionarse con el fenómeno migratorio.

Sin embargo, existen indicios que nos permiten afirmar precisamente lo contrario. Son pistas que nos hablan de movilidad y permanencia y, en efecto, de la posibilidad de encuadrar los programas en la sociología de las migraciones. El primer grupo de indicios tiene que ver con las solicitudes de reagrupamiento familiar cursadas al amparo de estos visados. Estas solicitudes pueden ser prudentemente interpretadas como un indicador de permanencia por parte del inversor, lo cual aproximaría los visados dorados, por sus efectos, al fenómeno migratorio. En el caso portugués, en 2013 se concedieron 576 reagrupamientos, 2.395 en 2014, 1.322 en 2015, 2.344 en 2016, 2.678 en 2017 y 2.264 hasta noviembre de 2018 (véase "Autorização de residencia para atividade de investimento (ARI)"). Con todo, desconocemos el número total de personas que se han establecido de forma efectiva en el país.

En el caso español, tan solo disponemos de datos agregados para el conjunto de los extranjeros que solicitan la residencia en España al amparo de la ley de los emprendedores (i.e., inversores, emprendedores, investigadores, profesionales altamente cualificados y trasladados intraempresariales). Así pues, es necesaria alguna precaución en su lectura, sobre todo si tenemos en cuenta que el número de reagrupaciones familiares de los ciudadanos inversores se encuentra integrado en los datos generales. En 2018 se registra un total acumulado de 12.568 permisos de residencia por reagrupación familiar, aunque esta cifra no permite distinguir la categoría con base en la cual se produce la reagrupación (véase Extranjeros residentes en España. A 30 de junio de 2018. Principales Resultados). Esto es, no sabemos si son familiares de inversores, emprendedores, investigadores, profesionales altamente cualificados o trasladados empresariales.

Un segundo grupo de indicios alude a la pluralidad de motivaciones que mueve a los solicitantes de los visados dorados. Contra cierto reduccionismo que ve en estas solicitudes motivos exclusivamente económicos, esto es, la maximización del retorno generado por la inversión, existen investigaciones que identifican motivaciones de naturaleza diversa que coexisten con las de cariz económico (Gaspar, 2017). Estos motivos pueden englobarse, genéricamente, bajo la etiqueta de migraciones por estilo de vida y se refieren a la movilidad vinculada a la búsqueda de una mayor calidad en el modo de vivir (Benson y O’Reilly, 2009) caracterizada por:

a) Tener como protagonistas a individuos con un poder adquisitivo significativo o considerablemente superior a la media.

b) Contener un proyecto vital que pretende ser desarrollado en contraposición a un modo de vida anterior que se entiende poco satisfactorio o directamente agotado.

c) Estar relacionada con valores post-materialistas (reflexividad, realización personal, renegociación del binomio tiempo laboral-tiempo individual, libertad, autonomía) integrados en un marco simbólico en el que la migración no es un acto y sí un proceso ligado a un nuevo comienzo.

d) Presentar niveles de autoempleo significativos, relacionados con la pretensión de gozar de autonomía propia y de independencia respecto a cualquier jerarquía laboral.

Estas características pueden ser encontradas en ciertos sectores de la sociedad china, origen del mayor número de golden visa en Portugal y España. Wong (2003) destaca este perfil de migración en el que, junto a las oportunidades de negocio, una creciente clase media se dirige a Australia, Canadá o Estados Unidos atraída por las posibilidades educativas para sus hijos, por unas condiciones de vida con mayor calidad y por la estabilidad legal y política de la que carecen en su país. Xu y Wu (2016) corroboran la pujanza de este perfil dentro de la propia China, materializado en jubilados, emprendedores y turistastrabajadores que se encaminan hacia las ciudades del sur huyendo de las grandes urbes y poseedores de algún volumen de capital con el que financiar su retiro o sus proyectos profesionales.

En Portugal este fenómeno también es constatable y se encuentra vinculado en parte al desarrollo del programa. En el estudio de Bongardt y Santos Neves (2014) sobre la comunidad china de negocios en territorio portugués los entrevistados mencionan otros motivos que justificarían su permanencia en el país: 
las oportunidades educativas y formativas para los más jóvenes, la estabilidad y la seguridad frente a los riesgos que comporta el sistema legal chino, las opciones de reagrupamiento familiar, la libertad de movimientos en el espacio Schengen, el capital simbólico acumulado en términos de prestigio basado en el contacto con la cultura occidental y europea, y un medio ambiente no sometido a los índices de contaminación y degradación de las ciudades chinas. Por lo tanto, es en este nicho donde con más claridad se observan los nexos entre visados dorados, migración y permanencia, y el que en consecuencia más potencialidades ofrece a la indagación sociológica.

En síntesis, los golden visa pueden constituir un objeto legítimo para la sociología si estos visados traen aparejadas ventajas de movilidad y permanencia (posibilidad verosímil si se atiende al reagrupamiento familiar o a la movilidad por estilo de vida que parecen practicar algunos de los más frecuentes beneficiarios).

\section{CONCLUSIÓN}

Los visados dorados son todavía una novedad en España y Portugal. Medidas tan recientes solo admiten una aproximación cautelosa a la espera de que su consolidación y evolución venga acompañada de más datos e información con los que ofrecer un análisis más completo. Lo que sabemos hasta aquí determina que estas conclusiones sean provisionales:

a) Conceptualmente entendemos que en España y Portugal los golden visa se encuadran en las migraciones de capital, con sus dos dimensiones asociadas: los programas de migración de negocios y la inversión directa extranjera. Esta última, como hemos señalado, no tiene por qué determinar necesariamente la movilidad geográfica y la permanencia del titular del visado.

b) En ambos países, los visados dorados se integran en la circulación mundial de flujos de capital y en las dinámicas competitivas que se establecen en pos de su atracción. Coyunturalmente estos programas nacen a raíz de la crisis de las deudas soberanas en Europa desde 2011, tras un periodo dominado por políticas de austeridad -con la reducción del gasto público y la contracción de la economía con vistas a su posterior expansión- en el que es estimulada la búsqueda de inversores que no generen una sobrecarga en el sistema de protección social.

c) Los visados dorados son el exponente de un tipo de racionalidad política neoliberal que propugna una noción de ciudadanía económica que justificaría el trato preferente a ciertos individuos como detentores de capital. Institucionalmente este tipo de ciudadanía es favorecida y modelada por el estado como pieza básica en la dinamización de un mercado de inversores extranjeros.

d) Los efectos cívico-políticos pasan por el riesgo de mercantilización de aspectos clave dentro del campo de la ciudadanía. Uno de estos aspectos es la residencia, que guarda relación con las ideas de comunidad, cohesión y participación. A su vez, esta ciudadanía modulada y articulada de acuerdo con las dinámicas del mercado introduce desigualdades en el trato del estado a los ciudadanos, basadas en el valor económico que porta cada individuo y que afectan negativamente al grado de legitimidad que los ciudadanos atribuyen a las instituciones.

e) En clave científica, estos programas pueden formar parte del campo de estudio de la sociología de las migraciones siempre que la inversión no venga acompañada de movilidad y permanencia. No obstante, existen indicios de que tales inversiones pueden vincularse a un tipo de migración por estilo de vida en la que, genéricamente, se pretende una mejoría cualitativa del modo de vivir.

Más allá de estas conclusiones existen cuestiones a la espera de investigación. En lo que toca a la descripción y al análisis de la evolución del fenómeno, los datos relativos a los visados dorados deben permitir un mayor nivel de desagregación (nacionalidades completas, zonas en las que se concentra la inversión, reproducción de la inversión, renovaciones, naturalización por vía de la ARI), amén de añadir información pertinente relacionada.

Con relación a los beneficiarios de los visados dorados, desconocemos quiénes son y cómo se articulan los motivos que condujeron a realizar su solicitud. Se hacen necesarias investigaciones cualitativas que den cuenta de las razones de aquellos beneficiarios y que permitan determinar hasta qué punto el marco de la migración por estilo de vida se ajusta a este tipo de movilidad y a sus protagonistas, así como los patrones de sedentarización y las estrategias de integración en el país de acogida.

En el futuro también será necesario determinar si el origen de los beneficiarios de los visados dorados se halla en colectivos con los que existe una relación importante en los ámbitos del turismo y de los negocios. 
Este análisis permitiría comprender mejor el alcance del programa y su contextualización en el marco de las relaciones internacionales de capital y del flujo de migraciones que mantienen España y Portugal con otros países.
Finalmente, dentro del contexto de recepción (países de acogida) necesitamos conocer las percepciones que los ciudadanos nacionales y la población inmigrante tienen del programa, lo que a su vez permitiría calibrar las consecuencias cívico-políticas del fenómeno.

\section{NOTAS}

[1] En este artículo emplearemos las dos expresiones indistintamente.

[2] En Portugal, la ARI estuvo precedida años antes por otra medida de carácter fiscal para facilitar la entrada de capital de ciudadanos extranjeros de cualquier nacionalidad: los Residentes No Habituales (RNH). Esta medida favorece la exención fiscal a jubilados y ciudadanos extranjeros altamente cualificados que no hayan residido en el país durante los cinco años previos a la solicitud. Esta exoneración fiscal se aplica a todos los beneficiarios extranjeros que se instalen oficialmente en Portugal y que posean fuentes de rendimiento con origen extranjero -depósitos bancarios de interés variable, plusvalías y alquileres de inmuebles, pensiones o empleo por cuenta propia. En el caso de rendimien- tos de trabajo dependiente y por cuenta propia altamente cualificados ejercidos en Portugal, la tasa fiscal única aplicable es del 20\%, independientemente del volumen de ingresos (Decreto-Lei no 249/2009, de 23 de septiembre) (Mendes, 2015; Mesquita, 2014).

[3] Ni España ni Portugal constituyen una excepción. En Europa países como Bulgaria, Chipre, Francia, Grecia, Irlanda, Hungría, Malta, Letonia y el Reino Unido aplican programas de residencia por inversión similares (Carrera, 2014; Mesquita, 2014; Mendes, 2015). Fuera de Europa existen también programas análogos con variaciones en los requisitos y el periodo de vigencia de los derechos de ciudadanía y residencia. Es el caso de Antigua y Barbuda, Dominica,
Granada, Australia, Nueva Zelanda, Canadá, Singapur y los Estados Unidos (Xu, El-Ashram y Gold, 2015, p. 5).

[4] Es el caso de Malta. El programa de inversión individual de Malta, desarrollado a finales de 2013, ha suscitado un amplio debate además de reproches por parte de la Comisión Europea y el Parlamento Europeo. En ese programa se concedía la nacionalidad maltesa a cualquier ciudadano de un país tercero que donara dinero al estado o invirtiera en la economía nacional (Carrera, 2014).

[5] Como sabemos, la propuesta de los 160.000 euros no prosperó y España fijó un montante similar al de sus competidores.

\section{BIBLIOGRAFÍA}

Alonso, L. E., Fernández Rodríguez, C. J. e Ibáñez Rojo, R. (2016). De la moral del sacrificio a la conciencia de la precariedad. Un análisis cualitativo de los discursos sobre la evolución de la crisis en España. Política y Sociedad, 53 (2), pp. 353-379. https://doi.org/10.5209/ rev_POSO.2016.v53.n2.49380

Ampudia de Haro, F. (2006). Administrar el yo: literatura de autoayuda y gestión del comportamiento y los afectos. $R e$ vista Española de Investigaciones Sociológicas, 113 (1), pp. 49-75. Disponible en http://www.reis.cis.es/REIS/PDF/ REIS 113 041168335219215.pdf

Ampudia de Haro, F. (2013). "Se não cuidarmos de nós, ninguém cuidará": Autoajuda financeira e racionalidade política neoliberal. Revista Crítica de Ciências Sociais, 101, pp. 111-134. https://doi. $\operatorname{org} / 10.4000 /$ rccs. 5386

Ampudia de Haro, F. (2014). El gobierno de la bancarrota: racionalidad neoliberal y educación financiera en Portugal. Papers. Revista de Sociología, 99 (3), pp. 1-18. https://doi.org/10.5565/rev/papers.609
Benson, M. y O’Reilly, K. (2009). Migration and the search for a better way of life: a critical exploration of lifestyle migration. Sociological Review, 57 (4), pp. 608-625. https://doi.org/10.1111/ j.1467-954X.2009.01864.x

Blyth, M. (2013), Austerity: The History of a Dangerous Idea. Nueva York: Oxford University Press.

Bongardt, A. y Santos Neves, M. (2014). The Chinese Business Community at a crossroads between crisis response and China's assertive global strategy. The case of Portugal. Migration Policy Center (MPC) Research Reports 2014/02. Disponible en http://cadmus.eui.eu/ bitstream/handle/1814/33142/MPC_ RR\%202014_02.pdf;sequence=1

Burchell, G., Gordon, C. y Miller, P. (eds.) (1991). The Foucault Effect: Studies in Governmentality. Londres: Harvester Wheatsheaf. https://doi.org/10.7208/ chicago/9780226028811.001.0001

Cabrerizo, M. (2013, 29 abril). El Gobierno eleva a 500.000 euros la inversión para

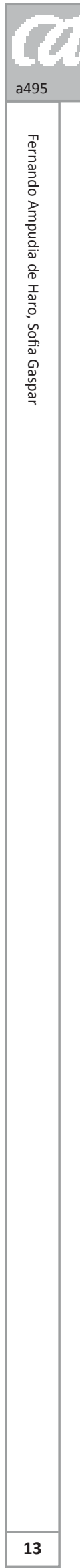

obtener la residencia. elEconomista.es. Disponible en https://goo.gl/EjHxLm

Carrera, S. (2014). How much does EU citizenship cost? The Maltese citizenshipfor-sale affair: A breakthrough for sincere cooperation in citizenship of the union? CEPS Paper in Liberty and Security in Europe, 64. Disponible en https://www. ceps.eu/publications/how-much-doeseu-citizenship-cost-maltese-citizenshipsale-affair-breakthrough-sincere

Dzankic, J. (2012). The Pros and Cons of lus Pecuniae: Investor citizenship in comparative perspective. EUI Working Papers RSCAS 2012/14. Robert Schuman Center for Advances Studies. EUDO Citizenship Observatory. Disponible en http:// cadmus.eui.eu/handle/1814/21476

Gaspar, S. (2017). Chinese migration to Portugal: Trends and Perspectives. Journal of Chinese Overseas, 13 (1), pp. 48-69. https://doi.org/10.1163/1793254812341343

Instituto Nacional de Estadística (2017). España en cifras 2017. Disponible en 
https://www.ine.es/prodyser/espa_cifras/2017/index.html\#1/z

Larner, W. (2000). Neo-Liberalism: Policy, Ideology, Governmentality. Studies in Political Economy, 63 (1), pp. 5-25. https://doi.org/10.1080/19187033.200 0.11675231

Marinis, P. de (1999). Gobierno, gubernamentalidad, Foucault y los anglofoucaultianos (Un ensayo sobre la racionalidad política del neoliberalismo). En Ramos Torre, R. y García Selgas, F. (eds.), Globalización, riesgo, reflexividad. Tres temas de la teoría social contemporánea. Madrid: Centro de Investigaciones Sociológicas, pp. 73-103.

Mendes, V. N. D. (2015). Incentivos ao Investimento Estrangeiro. O Regime Fiscal do Residente Não Habitual e a Autorização de Residência para Atividade de Investimento - Vistos Dourados (Golden Visa). [Tesis de maestría inédita]. Oporto: Instituto Superior de Contabilidade e Administração do Porto. Instituto Politécnico do Porto. Disponible en http://recipp. ipp.pt/bitstream/10400.22/6609/1/ DM_ValterMendes_2015.pdf

Mesquita, R. (2014). Portugal o novo "paraíso fiscal" para os estrangeiros - regime fiscal dos residentes não habituais e os golden visas. [Tesis de maestría inédita]. Lisboa: Universidade Católica Portuguesa. Disponible en http://repositorio.ucp. pt/handle/10400.14/16607

Ong, A. (2005). (Re)articulations of Citizenship. PS: Political Science and Politics, 38 (4), pp. 697-699. https://doi. org/10.1017/S1049096505050377

Ong, A. (2006). Neoliberalism as an exception. Mutations in citizenship and sovereignty. Durban, Londres: Duke University Press. https://doi. org/10.1215/9780822387879

Peláez-Paz, C. y Sanz Abad, J. (2014). Gestión diferencial de la población inmigrante y vulnerable: del discurso de la escasez a la ruptura de la universalidad de derechos. En Periferias, fronteras y diálogos. Actas del XIII Congreso de Antropología de la Federación de Asociaciones de Antropología. Tarragona: Universitat Rovira i Virgili, pp. 1201-1221.

Shachar, A. (2014). Dangerous Liaisons: Money and Citizenship. En Shachar, A. y Bauböck, R. (eds). Should Citizenship be for Sale? EUI Working Papers RSCAS 2014/01. Robert Schuman Center for Advances Studies. EUDO Citizenship Ob- servatory. Disponible en http://cadmus. eui.eu/bitstream/handle/1814/29318/ RSCAS 2014 01.pdf?sequence $=1$

Shachar, A. y Bauböck, R. (eds.) (2014). Should Citizenship be for Sale? EUI Working Papers RSCAS 2014/01. Robert Schuman Center for Advances Studies. EUDO Citizenship Observatory. Disponible en http://cadmus.eui.eu/bitstream/ handle/1814/29318/RSCAS_2014_01. pdf?sequence $=1$

Soler-Matutes, J. y Lijing, Y. (2012, 23 noviembre). Residencia a partir de 160.000 euros. Cinco Días. Disponible en http:// cincodias.com/cincodias/2012/11/23/ economia/1353911312_850215.html

Tseng, Y.-F. (2000). The Mobility of Entrepreneurs and Capital: Taiwanese Capital-Linked Migration. International Migration, 38 (2), pp. 143-168. https:// doi.org/10.1111/1468-2435.00105

Walsh, J. P. (2011). Quantifying citizens: neoliberal restructuring and immigrant selection in Canada and Australia. Citizenship Studies, 15 (6-7), pp. 861-879. https:// doi.org/10.1080/13621025.2011.600135

Wong, L. L. (2003). Chinese Business Migration to Australia, Canada and the United States: State Policy and the Global Immigration Marketplace. Asian and Pacific Migration Journal, 12 (3), pp. 301-336. https://doi. org/10.1177/011719680301200303

Xu, X., El-Ashram, A. y Gold, J. (2015). Too Much of a Good Thing? Prudent Management of Inflows under Economic Citizenship Programs. IMF Working Paper, WP/15/93. International Monetary Fund. Disponible en https://www. imf.org/external/pubs/cat/longres. aspx?sk=42884.0

Xu, H. y Wu, Y. (2016). Lifestyle mobility in China: context, perspective and prospects. Mobilities, 11 (4), pp. 509-520. https://doi.org/10.1080/17450101.201 6.1221027

\section{Otros recursos}

António Costa destaca política de alargamento dos "vistos gold" em visita à China. Observador, 9 octubre 2016. Disponible en http://observador. pt/2016/10/09/antonio-costa-destacapolitica-de-alargamento-dos-vistosgold-em-visita-a-china/

Autorização de residencia para atividade de investimento (ARI). Dados de 08 de outu- bro de 2012 a 30 de novembro de 2018 . Serviço de Estrangeiros e Fronteiras. Disponible en https://www.sef.pt/pt/Documents/Mapa_ARI_PT_novembro18.pdf

El Gobierno concederá la residencia a los extranjeros que compren una casa por más de 160.000 euros. Público, 19 noviembre 2012. Disponible en http:// www.publico.es/actualidad/gobiernoconcedera-residencia-extranjeros-compren.html

Extranjeros residentes en España. A 30 de junio de 2018. Principales Resultados. Ministerio de Trabajo Migraciones y Seguridad Social. Disponible en http:// extranjeros.mitramiss.gob.es/es/Estadisticas/operaciones/con-certificado/201806/Residentes_Principales_Resultados_30062018.pdf

Informe sobre la aplicación de la Sección de Movilidad Internacional de la Ley 14/2013, de 27 de septiembre, de apoyo a los emprendedores y su internacionalización. Abril 2015. Ministerio de Empleo y Seguridad Social, Ministerio de Asuntos Exteriores y Cooperación, Ministerio de Economía y Competitividad, Ministerio del Interior. Disponible en http://extranjeros.empleo. gob.es/es/UnidadGrandesEmpresas/ ley14_2013/documentacion/Informe_ anual_de_la_Seccion_de_Movilidad de_la_ley_14_2013.pdf

Los extranjeros obtendrán la residencia al comprar un inmueble de más de 500.000 €. El Mundo, 24 mayo 2013. Disponible en http://www.elmundo. es/elmundo/2013/05/24/suvivienda/1369402921.html

Paulo Portas diz que o programa que concede vistos 'gold' excedeu largamente o objetivo. RTP Notícias, 28 diciembre 2013. Disponible en http://www.rtp.pt/ noticias/economia/paulo-portas-dizque-o-programa-que-concede-vistosgold-excedeu-largamente-o-objetivo_ n706053

Paulo Portas: "Estamos disponíveis para melhorar sistema dos vistos gold". Público, 12 marzo 2015. Disponible en https://www.publico.pt/2015/03/12/ politica/noticia/paulo-portas-estamosdisponiveis-para-melhorar-sistemados-vistos-gold-1688971

Rajoy sobre la residencia para compradores: 'Tenemos que vender stock'. El Mundo, 19 noviembre 2012. Disponible en http:// www.elmundo.es/elmundo/2012/11/19/ espana/1353331380.html 
Relatório de Imigração, Fronteiras e Asilo. 2017. Serviço de Estrangeiros e Fronteiras. Disponible en https://sefstat.sef.pt/ Docs/Rifa2017.pdf

Vistos Gold. "Não é por haver uma fraude numa avaliação que se acaba com os exames", diz Portas. Diário de Notícias,
12 marzo 2015. Disponible en http:// www.dn.pt/politica/interior/vistosgold-nao-e-por-haver-uma-fraudenuma-avaliacao-que-se-acaba-com-osexames-diz-portas-4450033.html

Vistos gold: Quatro anos são positivos mas programa está longe de ser principal arma para captar investimento. $\mathrm{Ob}$ servador, 7 octubre 2016. Disponible en http://observador.pt/2016/10/07/ vistos-gold-quatro-anos-sao-positivosmas-programa-esta-longe-de-ser-principal-arma-para-captar-investimento/ 